\title{
Absorption and Emission Properties of Light Emitting Diode Structures Containing GaInN/GaN QWs
}

\author{
J. Binder ${ }^{a}$, K.P. Korona ${ }^{a}$, J. Borysiuk ${ }^{a}$, A. WysmoŁeK ${ }^{a}$, M. Baeumler ${ }^{b}, \mathrm{~K}_{\text {KÖHleR }}{ }^{b}$ \\ AND L. KIRSTE ${ }^{b}$ \\ ${ }^{a}$ Faculty of Physics, University of Warsaw, Hoża 69, 00-681 Warsaw, Poland \\ ${ }^{b}$ Fraunhofer Institute for Applied Solid State Physics, Tullastr. 72, 79108 Freiburg, Germany
}

\begin{abstract}
In this work we present measurements of GaInN/GaN light emitting diodes (LEDs) with an active layer consisting of three quantum wells made of $\mathrm{Ga}_{0.9} \mathrm{In}_{0.10} \mathrm{~N}$ that have different widths $(1.8 \mathrm{~nm}, 2.7 \mathrm{~nm}, 3.7 \mathrm{~nm})$. A comparison of emission and absorption (photocurrent) on the same sample revealed a shift in energy, with the emission energy being significantly lower. The shifts are about $0.02 \mathrm{eV}, 0.03 \mathrm{eV}$, and $0.04 \mathrm{eV}$ for the quantum wells having the widths of $1.8 \mathrm{~nm}, 2.7 \mathrm{~nm}$, and $3.7 \mathrm{~nm}$, respectively. This can be explained by a shift of the ground state energy caused by the quantum confined Stark effect. Calculations show that due to the spontaneous polarization and the piezoelectric effect a strong electric field of the order of $1 \mathrm{MV} / \mathrm{cm}$ was present in the GaInN quantum wells. Simulations of ground-state energies in the model of an infinite square well under the influence of an electric field with a matched effective well width were performed and used to interpret the experimental results.
\end{abstract}

PACS: 78.55.Cr, 72.40.+w, 73.61.Ey

\section{Introduction}

The technology of illumination is about to change towards an effective, energy saving future. Structures based on GaInN/GaN quantum wells (QWs) are playing an important role in the field of light emitting devices and a deeper understanding of the processes involved in light emission is therefore needed to further increase the capabilities of the produced structures.

Such GaInN/GaN quantum structures are also highly interesting because of their high built-in electric fields $[1,2]$ that lead to the observation of a significant quantum confined Stark effect (QCSE) [2-5]. This opens up a lot of possibilities concerning design and technology of such diode structures $[2,6-8]$, but also requires a profound knowledge of physical processes involved. The QCSE was mainly observed in emission spectra and simultaneous observations of absorption and emission are rare since absorption measurements of thin layer QWs are difficult. Below we present the comparison of luminescence and absorption that was measured by photocurrent spectroscopy.

\section{Samples}

The samples were grown by metal-organic chemical vapor deposition (MOCVD) on a sapphire substrate with a nucleation layer of $\mathrm{GaN}$, followed by a $n$-doped layer of GaN:Si. The active layer consists of three QWs made of $\mathrm{Ga}_{1-x} \mathrm{In}_{x} \mathrm{~N} / \mathrm{GaN}\left(x_{\text {In }}\right.$ about 0.10$)$ heterostructures followed by an $\mathrm{Al}_{x} \mathrm{Ga}_{1-x} \mathrm{~N}: \mathrm{Mg}$ barrier $\left(x_{\mathrm{Al}}\right.$ about 0.1$)$ and a $p$-doped layer of GaN:Mg. The three samples have different quantum well (QW) widths: $d=1.8 \mathrm{~nm}, 2.7 \mathrm{~nm}$, $3.7 \mathrm{~nm}$.
Contacts to the LEDs were made by pressing of indium or by evaporation of gold. Both techniques gave similar, good contacts ( $R$ about $100 \Omega$ ).

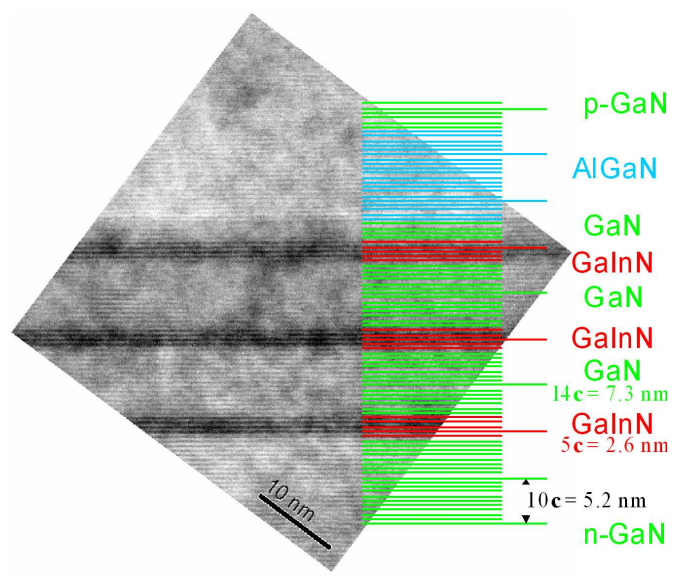

Fig. 1. Transmission electron microscope images of the LED structure grown on sapphire.

The microscopic structure of the $2.7 \mathrm{~nm}$ sample is presented in Fig. 1. The structure ideally fits to a scheme drawn taking into account the lattice parameter $c$ equal to $0.5185 \mathrm{~nm}, 0.4982 \mathrm{~nm}$, and $0.570 \mathrm{~nm}$ for GaN, AlN, and $\mathrm{InN}$, respectively [9].

\section{Quantum wells in an electric field}

Numerical calculations of the electric field and potential profiles were performed for this structure as described in [8]. It has been found that due to the spontaneous 
polarization and the piezoelectric effect $[1,2]$ a strong electric field was present in the GaInN QWs. This field has reverse direction compared to the field generated by the $p-n$ junction. The magnitude of the field is similar for all samples. It is about $0.8 \mathrm{MV} / \mathrm{cm}$ for zero bias and increases to $1.3 \mathrm{MV} / \mathrm{cm}$ at external bias of $3 \mathrm{~V}$.

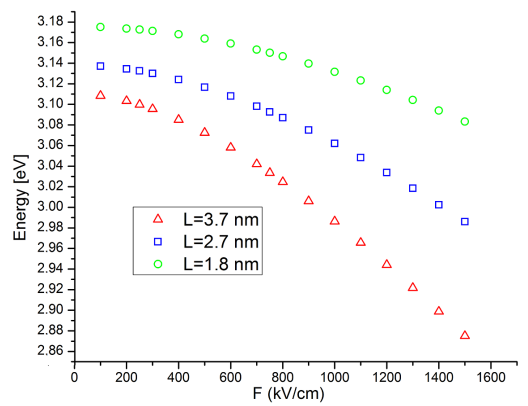

Fig. 2. Transition energies calculated for the three QWs with different electric fields.

In order to compare the experimentally obtained transition energies with theoretical expectations, we performed calculations for the quantum wells. We used the model of an infinite well under applied electric field. This model alone is not a good approximation for the ground states in the well because it does not take into account the impact of the finiteness of the confining potential and the resulting penetration of the wave function into the barriers. Therefore, we matched the ground state energy of an exactly calculated finite square well at zero field with an infinite square well obtaining an effective well width as proposed by Miller et al. [10, 11]. The effective well width is then used to calculate the wave functions and the ground state energies (Fig. 2) for electrons and holes in the model of an infinite well under influence of an electric field using

$$
\Psi(x)=a \operatorname{Ai}(x)+b \operatorname{Bi}(x)
$$

as wave function, where $\operatorname{Ai}(x), \operatorname{Bi}(x)$ are the Airy functions.

The obtained transition energies show a non-linear behavior (see Fig. 2) that is most pronounced for the widest QW as expected. The dependence of the energy in an electric field $F$ can be described as

$$
E(F)=E_{0}+p_{0} F+\beta F^{2},
$$

with $p_{0}$ being the initial dipole moment and $\beta$ - the system polarizability.

\section{Measurements and discussion}

In order to characterize the sample, measurements of photoluminescence (PL), electroluminescence (EL) and photocurrent (PC) were performed at room temperature. The PL was excited by the third harmonic of a Ti:sapphire laser tuned to be above AlGaN band gap. The PC was excited by a halogen lamp filtered by a monochromator. The emission was measured as a function of applied voltage, analyzed by a $320 \mathrm{~mm}$ spectrometer equipped with a CCD camera.

The voltage-current characteristics of the diodes show clearly exponential dependence.

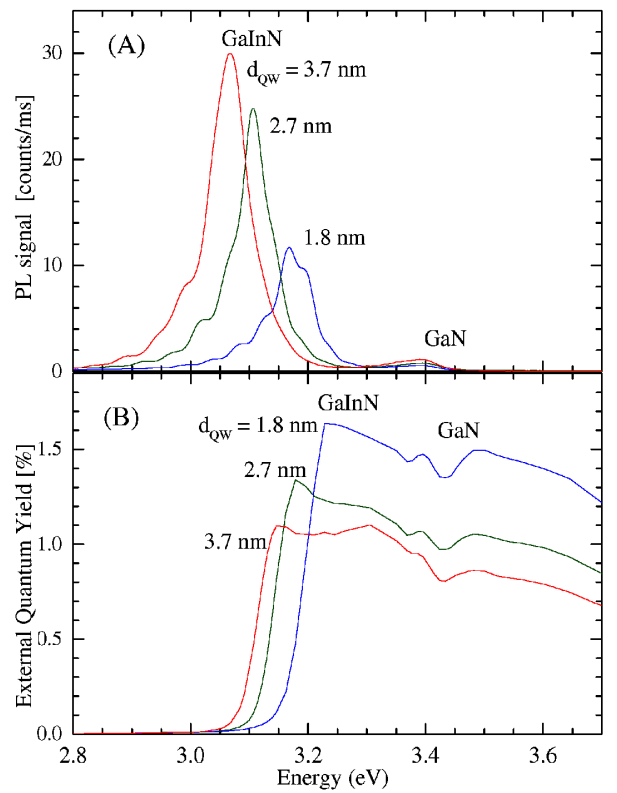

Fig. 3. Comparison of photoluminescence (A) and photocurrent (B) spectra. The peaks of the PL spectra are shifted to lower energies in respect of the corresponding thresholds of the PC spectra.

A strong emission of the QWs could be observed in EL at energies of $3.07 \mathrm{eV}, 3.10 \mathrm{eV}$, and $3.17 \mathrm{eV}$ for the $\mathrm{QWs}$ having the widths of $3.7 \mathrm{~nm}, 2.7 \mathrm{~nm}$, and $1.8 \mathrm{~nm}$, respectively. An additional peak from GaN was visible in PL at $3.4 \mathrm{eV}$ (Fig. 3A). The different QW widths resulted in different emission energies, which can be explained by different quantization energies and the presence of the internal electric field caused by spontaneous and piezoelectric polarizations. Comparing the measured transition energies with the calculated transitions we find a good agreement of energies for all three QWs for an internal field of about $0.5 \mathrm{MV} / \mathrm{cm}$, which is somewhat lower than the $0.8 \mathrm{MV} / \mathrm{cm}$ we obtained with self-consistent calculations. This deviation can be explained by an uncertainty in the In-content influencing strongly the band discontinuity and therefore our assumed well depths. Furthermore, we neglected the influence of the electric field of the $p-n$ junction outside of our QW in our transition energy calculations. Indeed, our self-consistent modeling shows that the wave functions are influenced by two electric fields of different sign since they are penetrating into the barriers.

The investigated diodes were biased up to $3.6 \mathrm{~V}$ in forward direction and down to $-3.5 \mathrm{~V}$ in reverse direction. At low or reverse bias only laser-excited photoluminescence was observed. At high positive bias electrolumines- 
cence was also present. Application of an external voltage leads to a change in the electric field of the QWs resulting in a shift of the ground state energies towards lower energies. However, the observed changes were relatively small. The strongest shift, $\Delta E=6 \mathrm{meV}$ was observed for the $3.7 \mathrm{~nm}$ sample. The shifts were lower than expected from the calculations probably because the electron wave function in the GaInN/GaN structures penetrates into the barriers and is therefore also influenced by an electric field of opposite direction compared to field in the well. Hence for the ground state energy calculations, one should take Eq. (2) using an effective electric field that is an average of the fields acting on the wave function $F_{\text {eff }}=\langle\Psi|F| \Psi\rangle /\langle\Psi \mid \Psi\rangle$.

The photocurrent measurements showed the presence of spectral bands related to the GaInN QWs, GaN and AlGaN (Fig. 3B). The external quantum efficiencies (ratio of the electric current in electrons/s to the incoming photon flux) were relatively high, of the order of $1 \%$. A comparison of the PC and the ellipsometric spectrum showed that the bands correspond strictly to the absorption bands, which was due to efficient delocalization of photoexcited carriers at room temperature. The energy thresholds of the QW absorption bands decreased with increasing QW width from $3.19 \mathrm{eV}$ for the $1.8 \mathrm{~nm} \mathrm{QW}$ to $3.11 \mathrm{eV}$ for the $3.7 \mathrm{~nm} \mathrm{QW}$, similarly to the case of emission.

The comparison of the emission (EL and PL) and absorption (PC) on the same sample revealed a shift in energy, with the emission energy being significantly lower (Fig. 3). The shifts were $16 \pm 10 \mathrm{meV}, 33 \pm 10 \mathrm{meV}$ and $42 \pm 10 \mathrm{meV}$ for the QWs having the widths of $1.8 \mathrm{~nm}$, $2.7 \mathrm{~nm}$, and $3.7 \mathrm{~nm}$, respectively. One should take into account that the emission originates from the ground state. For absorption, however, the ground states are not visible because of their low oscillator strength resulting from the separation of the electron and hole wave functions as a consequence of the electric field leading to a triangular character at the bottom of the QWs.

It has been observed that the shifts increase with increasing QW width. This can be explained using Eq. (2) since the dipole moment $p_{0}$ should be proportional to the QW width.

\section{Conclusions}

Three similar samples containing GaInN/GaN QWs with different well widths were measured in order to analyze the influence of an electric field on the electronic states in the wells. Several phenomena related to the QCSE were observed. Comparison of absorption and emission spectra revealed shifts towards lower energy in the case of emission, which can be explained by consid- ering that the emission originates from the lowest energy state. This state that is shifted to lower energy has a very low oscillator strength due to the QCSE and therefore cannot be observed in the absorption spectra.

The shifts in energy increased for wider QWs (from $0.02 \mathrm{eV}$ for the $1.8 \mathrm{~nm} \mathrm{QW}$ to $0.04 \mathrm{eV}$ for the $3.7 \mathrm{~nm}$ QW), which is in agreement with the numerical model taking into account that the electron wave function in the GaInN/GaN QWs penetrates into the barriers.

\section{Acknowledgments}

This work was partially supported by the Foundation for Polish Science International Ph.D. Projects Programme co-financed by the EU European Regional Development Fund andpartially supported by the JU ENIAC MERCURE project No. 120220.

\section{References}

[1] F. Bernardini, V. Fiorentini, D. Vanderbilt, Phys. Rev. B 56, R10024 (1997).

[2] O. Ambacher, J. Majewski, C. Miskys, A. Link, M. Hermann, M. Eickhoff, M. Stutzmann, F. Bernardini, V. Fiorentini, V. Tilak, B. Schaff, L.F. Eastman, J. Phys., Condens. Matter 14, 3399 (2002).

[3] D. Jena, S. Heikman, J.S. Speck, A. Gossard, U.K. Mishra, A. Link, O. Ambacher, Phys. Rev. B 67, 153306 (2003).

[4] K.P. Korona, A. Drabińska, K. Surowiecka, L. Wołowiec, J. Borysiuk, P. Caban, W. Strupiński, Acta Phys. Pol. A 114, 1179 (2008).

[5] M. Kunzer, C.-C. Leancu, M. Maier, K. Köhler, U. Kaufmann, J. Wagner, Phys. Status Solidi C 5, 2170 (2008).

[6] S. Nakamura, M. Senoh, S. Nagahama, N. Iwasa, T. Yamada, T. Matsushita, H. Kiyoku, Y. Sugimoto, T. Kozaki, H. Umemoto, M. Sano, K. Chocho, Appl. Phys. Lett. 72, 2014 (1998).

[7] K.P. Korona, A. Drabińska, A. Trajnerowicz, R. Bożek, K. Pakuła, J.M. Baranowski, Acta Phys. Pol. A 103, 675 (2003).

[8] K.P. Korona, A. Drabińska, P. Caban, W. Strupiński, J. Appl. Phys. 105, 083712 (2009).

[9] I. Vurgaftman, J.R. Meyer, L.R. Ram-Mohan, J. Appl. Phys. 89, 5816 (2001).

[10] D.A.B. Miller, D.S. Chemla, T.C. Damen, A.C. Gossard, W. Wiegmann, T.H. Wood, C.A. Burrus, Phys. Rev. Lett. 53, 2172 (1984).

[11] D.A.B. Miller, D.S. Chemla, T.C. Damen, A.C. Gossard, W. Wiegmann, T.H. Wood, C.A. Burrus, Phys. Rev. B 32, 1043 (1985). 